\title{
Chylothorax in a child with rifampicin-resistant tuberculosis
}

\author{
B McLaren, MB ChB, MMed (Paed), FC Paed (SA), Dip Allergy; X Song, MB ChB, FC Paed (SA); E Mate, MB ChB, FC Paed (SA); \\ C Jardine, BSc, MB BCh, FCPaed (SA), MMed (Paed); T Mabaso, BSc, MB ChB, FC Paed (SA), MMed (Paed); V Mammen, MB BCh, DCH, \\ FC Paed (SA), MMed (Paed), Cert Paed Cardiol; S Lala, MB BCh, FC Paed (SA), MMed (Paed), PhD; Z Dangor, MB BCh, FC Paed (SA), \\ MMed, PhD, Cert Paed Pulmonol; C Verwey, MB ChB, MMed (Paed), FC Paed (SA), Cert Paed Pulmonol
}

Department of Paediatrics and Child Health, Faculty of Health Sciences, University of the Witwatersrand, and Chris Hani Baragwanath Academic Hospital, Johannesburg, South Africa

Corresponding author: B McLaren (brittamclaren@gmail.com)

\begin{abstract}
Chylothorax is rare in children. Only a few cases of tuberculosis (TB)-associated chylothorax have been reported. We present a child on standard four-drug TB treatment who presented with wheezing and a chylothorax. Bronchoscopy showed caseating lymph nodes, and rifampicin-resistant TB was identified from the bronchoalveolar lavage specimen. There was marked clinical and radiological improvement 1 month after starting multidrug-resistant (MDR) TB treatment and steroids. The association of chylothorax and MDR-TB has not been described in children. MDR-TB should be considered in children who fail adherent, empirically started drug-susceptible TB treatment.
\end{abstract}

Afr J Thoracic Crit Care Med 2019;25(3):123-126. https://doi.org/10.7196/AJTCCM.2019.v25i3.237

The burden of pulmonary tuberculosis (PTB) is very high in subSaharan Africa, with a reported incidence of 500 per 100000 population. ${ }^{[1]}$ Children account for $\sim 15 \%$ of the total tuberculosis (TB) burden in developing countries. ${ }^{[2]}$ Culture-confirmed TB accounts for only $10-15 \%$ of children treated for TB. ${ }^{[3]}$ This is a result of the paucibacillary nature of PTB in children, and the fact that specimens are often not obtained. Drug susceptibility testing can only be done if bacteriological confirmation is achieved. In settings with a high incidence of drug-resistant TB, drug resistance should be considered in children with clinically diagnosed TB who have not improved, or have progressively worsened, despite adequate first-line TB therapy. Although drug-resistant TB has not been reported to be more virulent than drug-susceptible $\mathrm{TB},{ }^{[4]}$ the delay in identifying and treating these children may result in more advanced or disseminated disease.

Chylothorax is defined as a pleural effusion with triglyceride levels $>110 \mathrm{mg} / \mathrm{dL}$ and the presence of chylomicrons. ${ }^{[5]}$ It is hypothesised that TB lymph nodes erode through the thoracic duct, resulting in chylous fluid leaking into the thoracic space, of which only a few childhood cases have been reported. We describe a chylothorax in a child with rifampicin-resistant endobronchial TB.

\section{Case presentation}

An HIV-exposed uninfected 20-month-old boy presented to Chris Hani Baragwanath Academic Hospital in Johannesburg, South Africa, with a 2-day history of shortness of breath, cough and noisy breathing. He had also experienced night sweats, fever and vomiting for a week. Two weeks prior to this presentation, he had been diagnosed with bacteriologically unconfirmed PTB at a primary healthcare clinic and was empirically started on a four-drug antituberculosis treatment regimen (rifampicin, isoniazid, pyrazinamide and ethambutol). The diagnosis was based on a chest X-ray (Fig. 1A) and a positive Mantoux test. His father (a household contact) had been diagnosed with PTB, and had been on first-line antituberculosis treatment for 4 months.

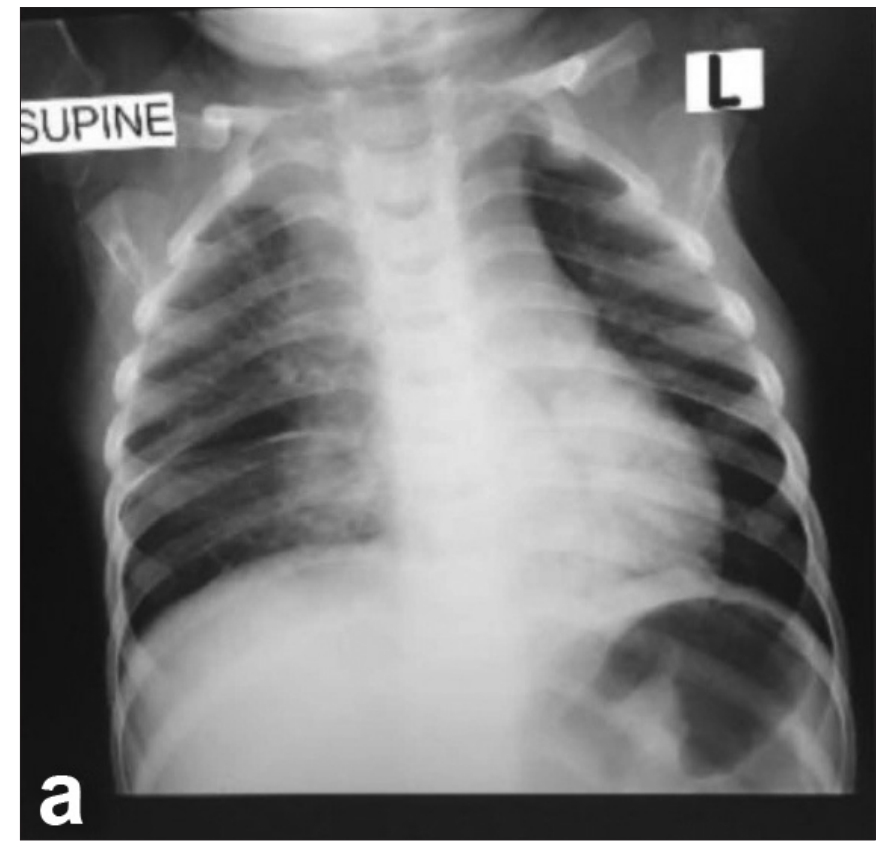

Fig. 1A. Chest X-ray on diagnosis of unconfirmed tuberculosis at the local clinic 1 month prior to index presentation, demonstrating attenuation of the trachea and right and left main bronchus.

The boy had had normal growth and development up to 1 year of age, but had subsequently lost weight, and fallen from a weight-for-height $z$-score +2 to the median over the preceding 8 months.

On admission, he was apyrexial, tachycardic and in moderate respiratory distress, with oxygen saturations of $97 \%$ in room air. $\mathrm{He}$ had marked chest wall indrawing and bilateral posterior cervical and axillary lymph nodes ( $<0.5 \mathrm{~cm}$ in diameter). He was clinically hyperinflated, with bilateral wheezing. The rest of his clinical examination was normal. 


\section{Case management}

He was diagnosed with viral bronchiolitis, and therefore a chest $\mathrm{X}$-ray was not performed. He was started on oxygen and hypertonic saline nebulisation. He responded poorly to this initial treatment and was therefore started on oral corticosteroids and amoxicillin on day 2 of hospitalisation, and his first-line antituberculosis treatment was continued. Gastric aspirate samples for microscopy for acid-fast bacilli, Xpert MTB/RIF (Cepheid, USA) and culture for mycobacteria were negative.

During his hospital admission period his wheezing persisted, and he remained in moderate respiratory distress. On the fifth day of admission, he developed percussion dullness and absent breath sounds over the right hemithorax.

A chest X-ray at this point revealed a large right-sided pleural effusion (Fig. 1B). A computed tomography scan of the chest revealed hilar lymphadenopathy and a large right-sided pleural effusion. The thoracic duct could not be visualised. Diagnostic pleurocentesis confirmed a chylothorax. It was suspected that tuberculous lymph nodes had eroded the thoracic duct causing the chylothorax, and therefore a bronchoscopy was performed, which revealed caseating lymph nodes in the right main bronchus suggestive of TB, and $90 \%$ obstruction of the left main bronchus. The Xpert MTB/RIF (Cephaid, USA) test on a bronchoalveolar lavage specimen identified rifampicinresistant Mycobacterium tuberculosis. Gastric aspirates, pleural fluid and bronchoalveolar lavage samples were all smear-negative for acidfast bacilli and mycobacterial culture-negative.

\section{Treatment}

The chylothorax was managed with a low-fat diet (with additional medium-chain triglycerides), therapeutic taps for worsening respiratory distress and an octreotide infusion. The boy was commenced on multidrug-resistant TB (MDR-TB) treatment (amikacin, levofloxicin, ethionamide, terizidone, pyrazinamide, ethambutol and high-dose isoniazid) and oral steroids.

\section{Outcomes and follow-up}

For further management, he was transferred to a hospital dedicated to the care of MDR-TB patients. One month later, he showed marked clinical improvement, with almost complete radiological resolution of the chylothorax, and reduced hilar lymphadenopathy (Fig. 1C). The full case overview is shown in Fig. 2.

\section{Discussion}

To our knowledge, this is the first reported case of a chylothorax in a child with drug-resistant PTB. We suspect that this child had had endobronchial PTB that had not responded to first-line TB therapy, and progressed to large airway compression and infiltration of the thoracic duct, resulting in chylous fluid leaking into the pleural space. Other possibilities to consider would be paradoxical enlargement of lymph nodes from partial TB treatment, as oral steroids were not initiated at the initial presentation to the primary healthcare clinic. ${ }^{[6]}$

In children, congenital malformations of the lymphatic system are the most common medical cause of chylothorax, although infrequent outside the neonatal period. ${ }^{[5]}$ Chylothorax due to malignancies is common in adults, but rare in children. ${ }^{[5]} \mathrm{TB}$-associated chylothorax has been described, but this is limited to case reports. A PubMed

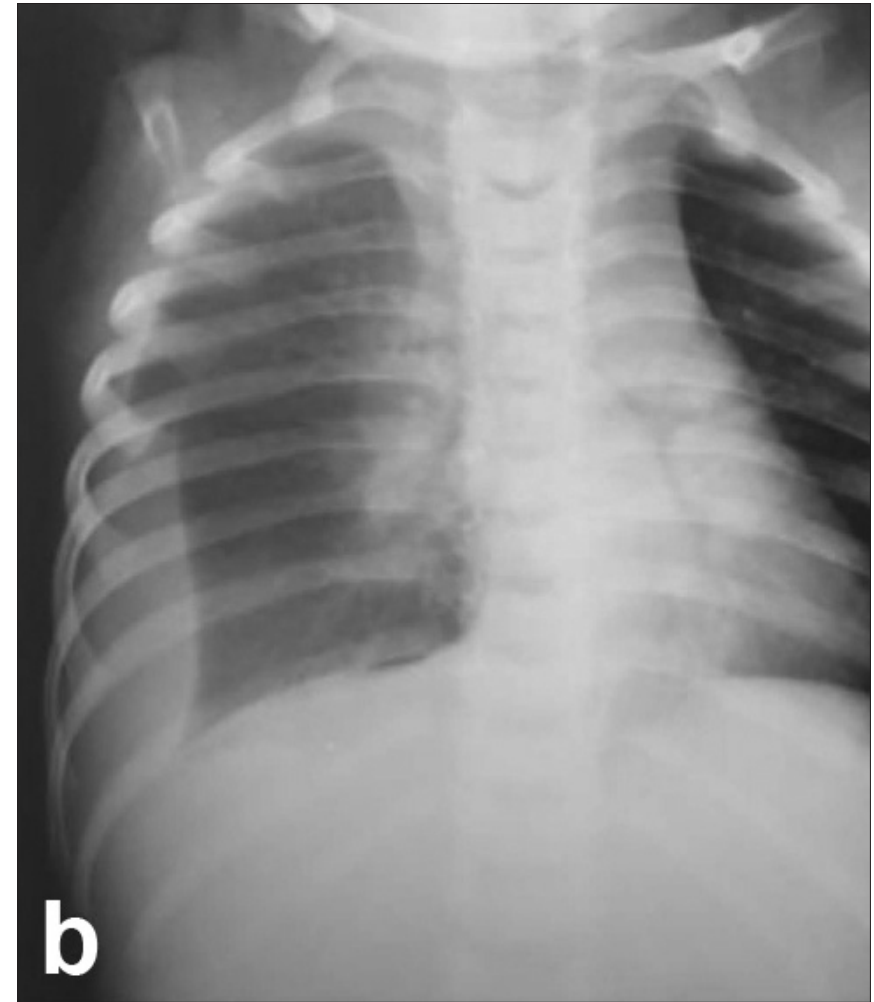

Fig. 1B. Chest X-ray on day 5 of admission, demonstrating right pleural effusion.

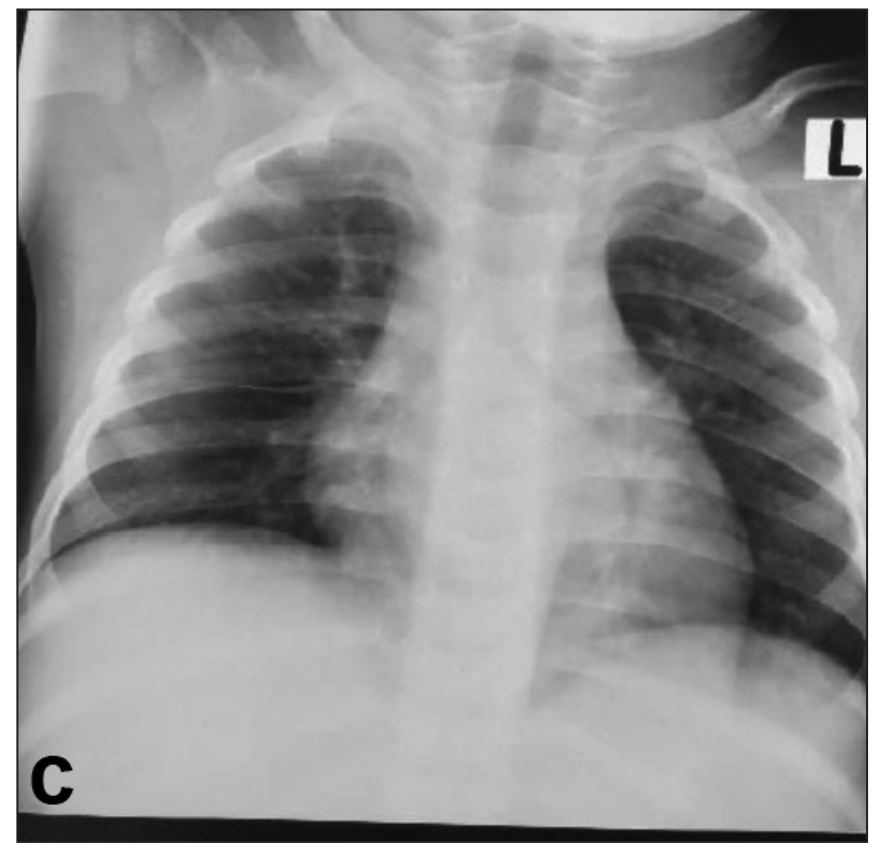

Fig. 1C. Chest X-ray after a month of rifampicin-resistant TB treatment, demonstrating radiological resolution of the chylothorax and loss of the attenuation of the trachea, right and left main bronchus.

search using the MeSH terms 'tuberculosis' and 'chylothorax' identified seven case reports of children with TB-associated chylothorax (age of presentation ranging from 4 months to 17 years). ${ }^{[7-12]}$ In three of the cases, TB was bacteriologically confirmed on gastric aspirate samples, in one case on bronchioalveolar lavage and in two cases on pleural fluid. All 


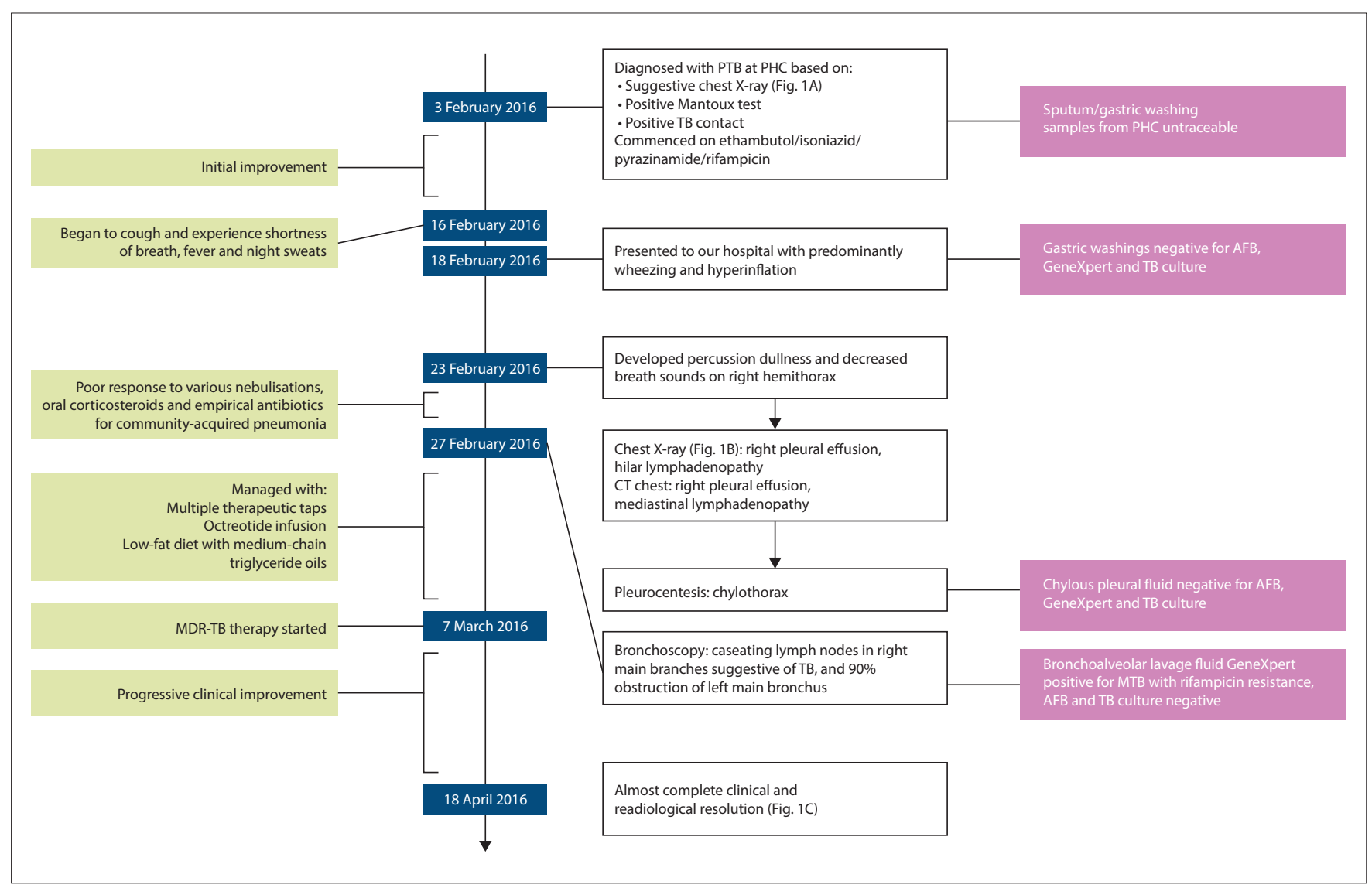

Fig. 2. Case overview. (MDR-TB = multidrug-resistant tuberculosis; PTB = pulmonary tuberculosis; $P H C=$ primary healthcare clinic; $C T$ chest $=$ computed tomography chest scan; $A F B=$ acid-fast bacilli; $M T B=$ Mycobacterium tuberculosis.)

cultures were susceptible to isoniazid and rifampicin. Most cases were associated with large TB perihilar lymphadenopathy. Our case differs in that the chylothorax was associated with drug-resistant TB. The delay in diagnosing drug-resistant TB and initiating appropriate second-line antituberculosis treatment may have led to progression of disease in this child, and this once again highlights the importance of vigorous contact screening, thorough clinical and microbiological investigation and initiation of appropriate antituberculosis treatment, as well as the implementation of a good follow-up plan for each case of TB.

\section{Conclusion}

This case highlights the difficulties in managing children diagnosed with unconfirmed TB, and the fact that drug-resistant TB must be considered in children who do not respond to conventional first-line TB treatment.

Although endobronchial TB is common in children, severe disease resulting in bronchial obstruction and progression to invasion of other mediastinal structures such as the thoracic duct is infrequent.

Acknowledgements. Informed consent to participate was obtained from the parents of the child presented in this case report. We thank the staff of Sizwe Tropical Disease Hospital, Johannesburg, for ongoing management of the case.

Author contributions. BM: conception and design, acquisition of case information, research and interpretation, drafting the article, critical revision and final approval of the version to be published; XS: conception and design, acquisition of case information, research and interpretation;
EM: conception and design, acquisition of case information, research and interpretation; CJ: acquisition of case information; TM: acquisition of case information; VM: acquisition of case information; SL: acquisition of case information, critical revision and final approval of the version to be published; ZD: conception and design, acquisition of case information, research and interpretation, drafting the article, critical revision and final approval of the version to be published; CV: conception and design, acquisition of case information, research and interpretation, drafting the article, critical revision and final approval of the version to be published, senior author.

Funding. None.

Conflicts of interest. None.

1. World Health Organization (WHO). Global tuberculosis report 2018. Geneva: WHO, 2018. https://www.who.int/tb/publications/global_report/en/ (accessed 2 February 2019).

2. Schaaf HS, Marais BJ, Whitelaw A, et al. Culture-confirmed childhood tuberculosis in Cape Town, South Africa: A review of 596 cases. BMC Infect Dis 2007;7:140. https:// doi.org/10.1186/1471-2334-7-140

3. Marais BJ, Pai M. New approaches and emerging technologies in the diagnosis of childhood tuberculosis. Paediatr Respir Rev 2007;8(2):124-133. https://doi. org/10.1016/j.prrv.2007.04.002

4. Fauci AS, NIAID Tuberculosis Working Group. Multidrug-resistant and extensively drug-resistant tuberculosis: The National Institute of Allergy and Infectious Diseases Research agenda and recommendations for priority research. J Infect Dis 2008;197(11):1493-1498. https://doi.org/10.1086/587904

5. Soto-Martinez M, Massie J. Chylothorax: Diagnosis and management in children. Paediatr Respir Rev 2009;10(4):199-207. https://doi.org/10.1016/j.prrv.2009.06.008

6. Singh A, Rahman H, Kumar V, et al. An unusual case of paradoxical enlargement of lymph nodes during treatment of tuberculous lymphadenitis in immunocompetent patient and literature review. Am J Case Rep 2013;14:201-204. https://doi. org/10.12659/ajcr.889013 
7. Grobbelaar M, Andronikou S, Goussard P, et al. Chylothorax as a complication of pulmonary tuberculosis in children. Pediatr Radiol 2008;38(2):224-226. https://doi. org/10.1007/s00247-007-0634-7

8. Rabie H, Lomp A, Goussard P, et al. Paradoxical tuberculosis associated immune reconstitution inflammatory syndrome presenting with chylous ascites and chylothorax in a HIV-1 infected child. J Trop Pediatr 2010;56(5):355-358. https://doi. org/10.1093/tropej/fmp141

9. Cakir E, Gocmen B, Uyan ZS, et al. An unusual case of chylothorax complicating childhood tuberculosis. Pediatr Pulmonol 2008;43(6):611-614. https://doi. org/10.1002/ppul.20817

10. Kim KJ, Park DW, Choi WS. Simultaneous chylothorax and chylous ascites due to tuberculosis. Infect Chemother 2014;46(1):50-53. https://doi.org/10.3947/ ic. 2014.46.1.50
11. Gie RP, Goussard P, Kling S, et al. Unusual forms of intrathoracic tuberculosis in children and their management. Paediatr Respir Rev 2004;5(Suppl A):S139-141. https://doi.org/10.1016/s1526-0542(04)90026-7

12. Kant S, Verma SK, Anand SC, et al. Development of bilateral chylothorax in a younger female secondary to tuberculosis. Lung India 2011;28(1):56-59. https://doi. org/10.4103/0970-2113.76303

Accepted 5 February 2019. 